\title{
SIR model with local and global infective contacts: A deterministic approach and applications
}

\author{
Alberto Maltz ${ }^{\mathrm{a}}$, Gabriel Fabricius ${ }^{\mathrm{b}, *}$ \\ a Departamento de Matemática, Facultad de Ciencias Exactas, Universidad Nacional de La Plata, CC 72, Correo Central, \\ 1900 La Plata, Argentina \\ ${ }^{\mathrm{b}}$ Instituto de Investigaciones Fisicoquímicas Teóricas y Aplicadas, Facultad de Ciencias Exactas, Universidad Nacional de La Plata, CC 16, Suc. 4, \\ 1900 La Plata, Argentina
}

\section{A R T I C L E I N F O}

\section{Article history:}

Received 20 May 2015

Available online 31 August 2016

\section{Keywords:}

Epidemics

SIR

Lattice

Deterministic model

Pair approximation

Pertussis

\begin{abstract}
A B S T R A C T
An epidemic model with births and deaths is considered on a two-dimensional $L \times L$ lattice. Each individual can have global infective contacts according to the standard susceptible-infected-recovered (SIR) model rules or local infective contacts with their nearest neighbors. We propose a deterministic approach to this model and, for the parameters corresponding to pertussis and rubella in the prevaccine era, verify that there is a close agreement with the stochastic simulations when epidemic spread or endemic stationarity is considered. We also find that our approach captures the characteristic features of the dynamic behavior of the system after a sudden decrease in global contacts that may arise as a consequence of health care measures. By using the deterministic approach, we are able to characterize the exponential growth of the epidemic behavior and analyze the stability of the system at the stationary values. Since the deterministic approximation captures the essential features of the disease transmission dynamics of the stochastic model, it provides a useful tool for performing systematic studies as a function of the model parameters. We give an example of this potentiality by analyzing the likelihood of the endemic state to become extinct when the weight of the global contacts is drastically reduced.
\end{abstract}

(C) 2016 Elsevier Inc. All rights reserved.

\section{Introduction}

Mathematical modeling of infectious diseases has become an area of increasing interest in recent decades (Anderson and May, 1991; Keeling and Rohani, 2008; Heesterbeek et al., 2015). Mathematical models are powerful tools for understanding the complex problem of infectious disease transmission. After the success of the simple SIR compartmental model in the description of the basic and common features of the transmission process (Kermack and McKendrick, 1927; Anderson and May, 1991), models have become more complex and specific for different infectious diseases in order to help in the evaluation and design of control strategies (Hethcote, 1997, 1999; Granich et al., 2009; Fabricius et al., 2013). These complexities may include age structure of the population, immune status of the individuals, structure of the social contacts and spatial heterogeneity.

\footnotetext{
* Corresponding author.

E-mail addresses: alberto@mate.unlp.edu.ar (A. Maltz), fabricius@fisica.unlp.edu.ar (G. Fabricius).
}

Wherever possible, deterministic compartmental models are usually chosen because it is easy to solve them numerically, interpret their results directly, and gradually increase their complexity by adding new compartments. However, we have known the importance of stochastic effects from the beginning of infectious disease modeling (Bartlett, 1956). Recent work has highlighted the significance that a stochastic treatment could have in the transmission of some diseases such as pertussis (Rohani et al., 1999; Bauch and Earn, 2003). In particular, the randomness and heterogeneity of contacts are some of the intrinsically stochastic aspects of the contagion process and knowing the accuracy that can be obtained with a deterministic approach to the problem is not a trivial matter. This kind of exploration is one of the purposes of the present work.

The heterogeneity of contacts in epidemic models is usually modeled by networks that range from the structureless random networks (Keeling and Eames, 2003) or the simple square lattice (Souza and Tomé, 2010) to more sophisticated networks where a local household structure is combined with random contacts (Ball et al., 1997, 2010), bipartite networks including information from actual census and population-mobility data (Eubank et al., 2004), or dynamical networks where the available connections of each node may change with time (for a recent 
review on epidemics on networks see Pastor-Satorras et al., 2015). In order to approximately treat the dynamics of different epidemic models on networks, some authors have taken a pair of nodes as their basic variables to construct Pair-Wise Models for networks (Keeling and Rohani, 2008). The transmission dynamics are then described by systems of ordinary differential equations. In a previous work, Joo and Lebowitz (2004) considered a susceptible-infected-recovered-susceptible (SIRS) spatial stochastic model having only local infective contacts on the hypercubic lattice and developed a pair approximation scheme to approximate the dynamics by a system of ordinary differential equations.

In the present work, we study a stochastic model on a lattice proposed by Verdasca et al. (2005) where local and global contacts are considered by emulating the situation of social relations where some "fixed" people are frequently contacted (the neighbors on the lattice) and some other unknown people are met by chance (the global contacts). In this model, which is inspired in the small-world network proposed by Watts and Strogatz (1998), a parameter $p$ is introduced to characterize the weight of global random contacts relative to local contacts. In this way, we can systematically analyze how the relative importance of the two contact types affects the disease transmission dynamics. Other authors have studied the stochastic fluctuations of this model for different values of $p$ and model parameters that include several infectious diseases (Simöes et al., 2008). Dottori and Fabricius (2015) studied the quasi-stationary state of the model for parameters corresponding to pertussis and quantified the relation between the effective transmission of the disease and the correlation of susceptible individuals with their infected neighbors.

We propose a deterministic approximation to the stochastic model with the local and global contacts mentioned above, where the local contacts on the square lattice are treated here under a pair approximation scheme based on the one used by Joo and Lebowitz (2004). For an introduction to the pair approximation in lattices, see Ellner (2001). We compare the predictions of our proposed deterministic approximation with the results of simulations performed with the stochastic model for parameters corresponding to two infectious diseases: pertussis and rubella. We make this comparison for different situations of the disease transmission dynamics: the epidemic spread, the endemic state of the disease, and dynamic perturbations that may arise from implemented control measures against the disease. Given the inability of the deterministic approach to account for the heterogeneity of the local configurations present in the stochastic model, it is not obvious a priori whether these heterogeneities could play a decisive role in some of the considered scenarios. However, in all the cases studied, the results obtained from the stochastic model simulations are closely approximated by the deterministic approach. Beyond this validation of the deterministic approximation, in this paper we present additional benefits to be gained from using this simpler approach.

\section{The stochastic model}

We consider the stochastic model studied by Dottori and Fabricius (2015). Individuals are identified with sites of an $L \times L$ square lattice under periodic conditions. Each one of the $N=L^{2}$ individuals may be in one of the three epidemiological states: $S, I$ or $R$ (susceptible, infected or recovered). The dynamics of the model are described by a stochastic Markovian process in which an individual may undergo one of the following changes in its state: $S \rightarrow I$ (infection), $I \rightarrow R$ (recovery), $S \rightarrow S, I \rightarrow S, R \rightarrow S$ (death and birth). Infections occur through infective contacts among susceptible and infected individuals. We define an infective contact as a contact between two individuals such that if one individual is susceptible and the other infected, the former becomes infected. We assume that an individual at a given site has an infective contact with a randomly chosen individual on the lattice with transition rate $p \beta$, and with one of its four nearest neighbors with transition rate $(1-p) \beta$. Local contacts represent the contacts in the circle of stable relations of an individual, while global random contacts represent people met by chance (for example, on a bus, at the supermarket, etc.). By changing $p$, we may change the relative weight of the global and local contacts in the system. The case $p=1$ corresponds to the classical SIR model (uniform mixing) where an individual may have an infective contact with any other individual in the system with the same transition rate $\beta$. On the other hand, the case $p=0$ corresponds to the square lattice where an individual may only contact one of its four nearest neighbors. Recovery from infection in this model is the same for every site and occurs at a transition rate $\gamma$. Deaths are assumed to be independent of the individual's state and occur at the same transition rate $\mu$. When an individual dies at a site, another individual is born simultaneously at this site in order to avoid empty sites. We assume that all newborns are susceptible individuals. Stochastic simulations are performed using the Gillespie algorithm (Gillespie, 1976). For a detailed description of the model and the implementation of the simulation algorithm, see Dottori and Fabricius (2015).

\section{A deterministic approach}

We consider an $L \times L$ lattice with periodic boundary conditions. At each time $t$, the individuals must be in one of the three states: $S$, $I$ or $R$ (susceptible, infected or recovered). We define $C_{s}, C_{i}$ and $C_{r}$ as the total number of individuals in those states. We denote $U_{a}=H$ when the state of the individual $a$ is $H$ (for example, $U_{a}=S$ ). Each pair $a b$ of (horizontal or vertical) neighboring individuals can be in six possible states: $S S, S I, S R, I I, I R, R R$. We remark that in both cases, $\left(U_{a}=S, U_{b}=I\right)$ and $\left(U_{a}=I, U_{b}=S\right)$, the pair $a b$ is in state $S I$ (and the same holds for $S R$ and $I R$ ). Let $C_{s s}, C_{s i}, C_{s r}, C_{i i}, C_{i r}, C_{r r}$ be the total number of each pair type of neighboring individuals. We define the unknowns $X_{s}, X_{i}, X_{r}, X_{s s}, X_{s i}, X_{s r}, X_{i i}, X_{i r}$ and $X_{r r}$ as the preceding quantities normalized by $N$.

They are functions of the time $t$ that are linked by

$$
\begin{aligned}
& X_{s}+X_{i}+X_{r}=1, \\
& X_{s s}+X_{s i}+X_{s r}+X_{i i}+X_{i r}+X_{r r}=2, \\
& X_{s}=\frac{2 X_{s s}+X_{s i}+X_{s r}}{4} \text { and } \\
& X_{i}=\frac{2 X_{i i}+X_{s i}+X_{i r}}{4} .
\end{aligned}
$$

In view of the above considerations:

(1) and (2) hold because there are $N$ individuals and $2 N$ pairs of neighboring individuals (remember that we have periodic conditions in the boundary). To obtain (3) (and analogously (4)) we consider that each individual in susceptible state $S$ belongs to four pairs of neighboring individuals of type SS, SR or SI but in their total contribution $4 C_{S}$, the $S S$ pairs are counted twice (remember the proportionality between $X_{s}, X_{s i}, .$. and $\left.C_{s}, C_{s i}, ..\right)$.

The pair approximation treatment of local infective contacts follows Joo and Lebowitz (2004) where an SIRS model with only local contacts is considered. It is basically a moment closure method that stops at the second moment. We describe below its basic idea.

Let $a b$ and $b c$ be two different pairs of neighboring individuals sharing $b$. The key point consists in the approximation of the probability that the triplet $a b c$ reaches a given state $\left(U_{a}=H_{1}\right.$, 


$$
\begin{aligned}
& \left.U_{b}=H_{2}, U_{c}=H_{3}\right) \text { at time } t \text { by } \\
& P_{t}\left(U_{a}=H_{1}, U_{b}=H_{2}, U_{c}=H_{3}\right) \\
& \simeq \frac{P_{t}\left(U_{a}=H_{1}, U_{b}=H_{2}\right) P_{t}\left(U_{b}=H_{2}, U_{c}=H_{3}\right)}{P_{t}\left(U_{b}=H_{2}\right)} .
\end{aligned}
$$

In order to apply (5) to the treatment of the local contacts in the deterministic approximation, we consider the natural correspondence between fractions (our unknowns in the deterministic approximation) and probabilities. For example $X_{s}$ (the fraction of susceptible individuals at time $t$ in the deterministic approximation) can be regarded as the probability that a given individual $a$ is of type $S$ at time $t$ in the stochastic model and $X_{s i} / 2$ (the fraction of $S I$ pairs at time $t$ in the deterministic approximation) as the probability that a given pair $a b$ of neighboring individuals is of type $S I$ at time $t$ in the stochastic model.

In the equations that we propose, the terms related to the birth-death and recovery rates do not depend on the local structure and are treated as in the classic SIR scheme. We have different transfer rates $r$ between classes due to infections. For each one of to the cases $(S \rightarrow I, S S \rightarrow S I, S I \rightarrow I I S R \rightarrow I R)$ we know the transfer rates $r_{1}$ and $r_{2}$ for local and global infections, respectively. Then, according to the stochastic model rules we take the transfer rate $r=: q r_{1}+p r_{2}$, where $q=1-p$.

We present now a characteristic case to show how the preceding observations are used (we omit the details of the whole construction of the system because the procedure for the other terms is similar).

Let $r$ be the rate at which $X_{i i}$ grows at the expense of the decrease of $X_{s i}$. For global contacts, $r_{2}=\beta X_{i}$. Let $a b$ be a pair of type $S I$ at a fixed time $t$ such that $U_{a}=I, U_{b}=S$. The rate of local infection for this pair is the product of $\beta$ and the fraction of infected individuals in the four nearest neighbors of $b$. This fraction can be regarded as $\left(1+3 p_{1}\right) / 4$, where $p_{1}$ is the probability that a fixed triplet $a b c$ ( $c$ being a fixed nearest neighbor of $b$ different from $a$ ) is in situation $\left(U_{a}=I, U_{b}=S, U_{c}=I\right)$, given that $\left(U_{a}=I, U_{b}=S\right)$. We have

$p_{1}=\frac{P_{t}\left(U_{a}=I, U_{b}=S, U_{c}=I\right)}{P_{t}\left(U_{a}=I, U_{b}=S\right)}$.

Then, using (5) and $P_{t}\left(U_{a}=I, U_{b}=S\right)=P_{t}\left(U_{a}=S, U_{b}=I\right)$,

$p_{1} \simeq \frac{P_{t}\left(U_{a}=S, U_{b}=I\right)}{P_{t}\left(U_{a}=S\right)}$,

which corresponds to $X_{s i} / 4 X_{s}$ in the deterministic approximation. Then we take

$r_{1}=\beta\left(\frac{3 X_{s i}}{16 X_{s}}+\frac{1}{4}\right)$

and $\left(q r_{1}+p r_{2}\right) X_{s i}$ is incorporated as a subtracted term in Eq. (10) and as an added term in Eq. (12) of the system that we present below.

$$
\begin{aligned}
\frac{d X_{s}}{d t}= & -p \beta X_{s} X_{i}-\frac{q \beta X_{s i}}{4}+\mu X_{i}+\mu X_{r}, \\
\frac{d X_{i}}{d t}= & -\gamma X_{i}-\mu X_{i}+p \beta X_{s} X_{i}+\frac{q \beta X_{s i}}{4}, \\
\frac{d X_{r}}{d t}= & -\mu X_{r}+\gamma X_{i}, \\
\frac{d X_{s s}}{d t}= & -2 p \beta X_{i} X_{s s}-\frac{3 q \beta X_{s i} X_{s s}}{8 X_{s}}+\mu X_{s i}+\mu X_{s r}, \\
\frac{d X_{s i}}{d t}= & -p \beta X_{i} X_{s i}-q \beta\left(\frac{3\left(X_{s i}\right)^{2}}{16 X_{s}}+\frac{X_{s i}}{4}\right)-\gamma X_{s i}-\mu X_{s i} \\
& +2 p \beta X_{i} X_{s s}+\frac{3 q \beta X_{s i} X_{s s}}{8 X_{s}}+2 \mu X_{i i}+\mu X_{i r},
\end{aligned}
$$

$$
\begin{aligned}
\frac{d X_{s r}}{d t}= & -p \beta X_{i} X_{s r}-\frac{3 q \beta X_{s i} X_{s r}}{16 X_{s}}-\mu X_{s r} \\
& +\gamma X_{s i}+\mu X_{i r}+2 \mu X_{r r}, \\
\frac{d X_{i i}}{d t}= & -2 \mu X_{i i}-2 \gamma X_{i i}+p \beta X_{i} X_{s i}+q \beta\left(\frac{3\left(X_{s i}\right)^{2}}{16 X_{s}}+\frac{X_{s i}}{4}\right), \\
\frac{d X_{i r}}{d t}= & -2 \mu X_{i r}-\gamma X_{i r}+2 \gamma X_{i i}+p \beta X_{i} X_{s r}+\frac{3 q \beta X_{s i} X_{s r}}{16 X_{s}} \\
\frac{d X_{r r}}{d t}= & -2 \mu X_{r r}+\gamma X_{i r}
\end{aligned}
$$

By using equalities (1)-(4) we reduce the system to five equations to perform our numerical calculations. In Appendix we make some considerations about this system.

\section{Results and discussion}

In this section we compare the results obtained from the stochastic model simulations with the results obtained using the deterministic approximation. In Section 4.3 .2 we present a study that involves only the deterministic approximation.

We consider $L=800$, which mimics a city of $N=640,000$ inhabitants. For pertussis we take $\mu=1 /(50$ years $), \gamma=$ $1 /$ (21 days) and $\beta=0.81 /$ day, which are standard parameters for SIR description of the disease in the pre-vaccine era (Rozhnova and Nunes, 2012). For rubella we take $\mu=1 /$ (50 years), $\gamma=1 /$ (18 days) and $\beta=0.3891 /$ day (Keeling et al., 2001). In order to choose the range of $p$ to be studied, we first estimate the basic reproductive ratio $R_{0}$ for the stochastic model, which is defined as the average number of secondary infections that an infected individual produces in the population when all the other individuals are susceptible (Anderson and May, 1991; Diekmann et al., 2013). $R_{0}$ is a widely used parameter because $R_{0}=1$ gives a threshold above which the disease is expected to spread in the system and below which it is expected to die out. The analytical computation of $R_{0}$ is not an easy task even for $p=0$, as has already been noted by other authors in similar models (Aparicio and Pascual, 2007). We compute $R_{0}$ numerically for the stochastic model and obtain that for $p=0$ it approaches 2.77 for pertussis and 2.34 for rubella, being an increasing function of $p$. Therefore, for the two problems to be studied, the disease could in principle be established in the stochastic model for any value of $p$. However, in this study we consider values of $p \geq 0.2$ since Dottori and Fabricius (2015) observed that for lower values of $p$ the probability of establishment and survival of the steady state for the stochastic model (representing the endemic state of pertussis in the prevaccine era) is very low because of fluctuations (see Section 4.2). In the case of rubella, this probability is low even for $p=0.2$. In this work, we are interested in considering values of the parameters that may represent real systems; however, we include the case $p=0.2$ for rubella for comparison purposes.

In the deterministic approach, differential equations are integrated using the Euler method with a time step of 0.01 days.

\subsection{Epidemic behavior}

We first study the epidemic spread of the disease for the case $p=0.4$. We consider the evolution of the system when a single infected individual is introduced in a population of $N-1$ susceptible individuals. From these initial conditions, 5000 independent runs are performed for the stochastic model with different sets of random numbers. For the deterministic approach we take $X_{s}=1-1 / N, X_{i}=1 / N, X_{s s}=2-4 / N, X_{s i}=4 / N$, and everything else 0 as initial conditions. 

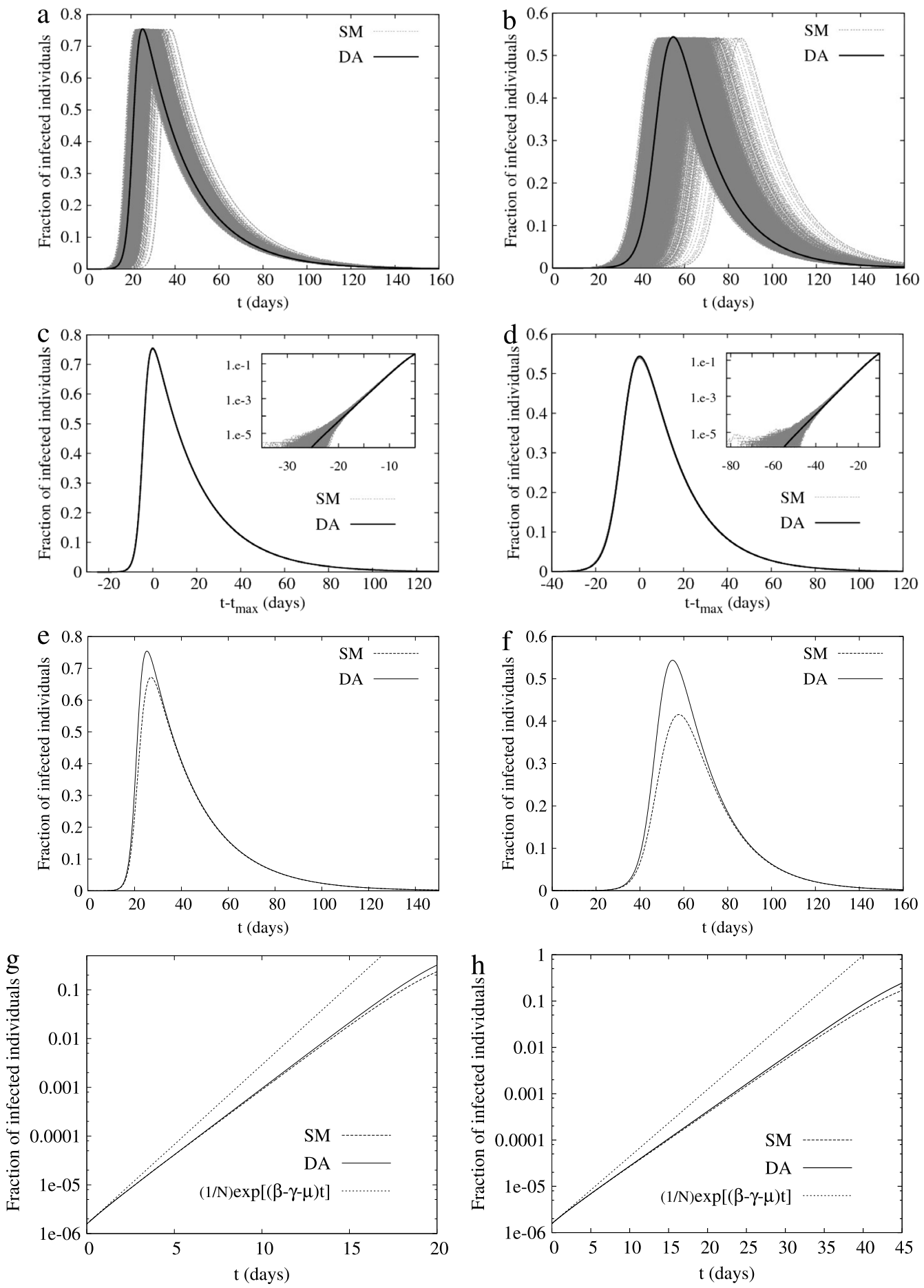

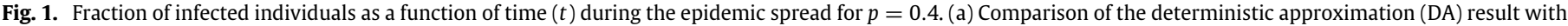

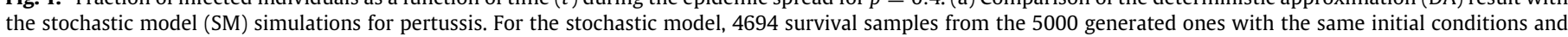

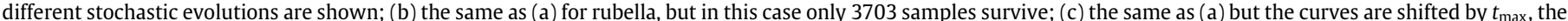

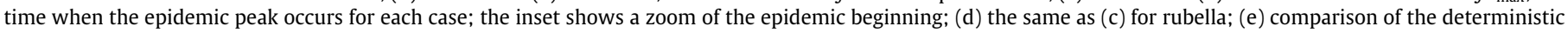

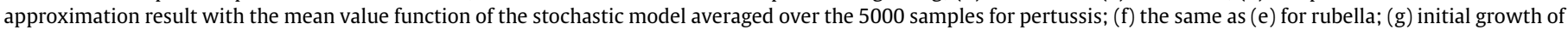
the epidemic for pertussis (details of figure (e)); (h) initial growth of the epidemic for rubella (details of figure (f)).

Because in the stochastic model there is a single infected individual at the beginning, there is a chance that it will recover before infecting anyone else, and the system will become extinct before developing the epidemic peak. In Fig. 1(a) and (b) we compare, for pertussis and rubella, the dynamical evolution of the survival samples for the stochastic model with the deterministic approximation results. There is a large dispersion of the time at which the epidemic peak occurs for the different stochastic model sam- ples, but the height of the peak is quite regular and close to the one obtained with the deterministic approximation. To analyze the evolution of the epidemic development independently of the time when the peak is reached, in Fig. 1(c) we plot the same curves as in Fig. 1(a), but shifting them rigidly such that all the maxima occur at the same time. The almost perfect collapse obtained for all the samples when the fraction of infected individuals is greater than 0.001 indicates that stochasticity influences the beginning of the process, 
but when the epidemic regime is established, the dynamical evolution is slightly influenced by stochastic effects. The same analysis is valid for the case of rubella in Fig. 1(d), where the deterministic approximation curve is hardly distinguishable from the collapsed stochastic model sample curves in the linear scale plot.

In Fig. 1(e) and (f) we compare again the result for the deterministic approximation but now with the average of the 5000 runs of the stochastic model simulations. The discrepancy in the maximum of the fraction of infected individuals in this case is because in the average for the stochastic model runs we include the extinctions. With the purpose of analyzing in more detail the beginning of the epidemic spread, in Fig. 1(g) and (h) we plot a detail of Fig. 1(e) and 1f on a logarithmic scale. At the first step of the dynamics, the value of $p$ is irrelevant as the infected individual can only contact susceptible ones regardless of whether the contacts are local or global. This can be seen in Fig. 1(g) and $1 \mathrm{~h}$, where at $t=0$ the stochastic model simulations and the deterministic approximation predict an exponential increase in the fraction of infected individuals at a rate $\beta-\gamma-\mu$, that is, 0.752 $1 /$ day for pertussis and 0.3331 /day for rubella. This is the behavior expected for the classical SIR model with the corresponding $\beta, \gamma, \mu$ parameters. To obtain this agreement between the averages over the different stochastic model samples and the deterministic approximation result at the very beginning of the epidemic spread, it is necessary to consider all the samples, even those in which the recovery of the infected individual occurs before the contagion to other susceptible individuals; if only survival samples were kept, the stochastic model averages would give an initial rate greater than $\beta-\gamma-\mu$. An interesting result predicted by the stochastic model simulations (and closely described by the deterministic approach) is the approximately exponential growth of the epidemic a few days after its beginning with an exponent that differs from the initial one. This can be concluded from the linear behavior observed in the curves of Fig. 1(g) from 5 to 15 days, and in Fig. 1(h) from 10 to 30 days. Exponential fits, $c \exp (\epsilon t)$, to the curves in the mentioned ranges give exponents $\epsilon_{\mathrm{SM}}=0.615$ and $\epsilon_{\mathrm{DA}}=0.626$ for pertussis, and $\epsilon_{\mathrm{SM}}=0.267$ and $\epsilon_{\mathrm{DA}}=0.272$ for rubella, where the subscripts SM and DA refer to the stochastic model and the deterministic approximation, respectively.

In order to characterize this behavior as a function of $p$ in this exponential regime, the exponents could be written as: $\epsilon(p)=$ $b(p)-\gamma-\mu$. That is to say, the same as in the classical SIR model (where only global contacts are present) but with an effective contact rate at the epidemic growth, $b(p)$, that is different from $\beta$ because of the presence of local contacts. So, we define

$b_{\mathrm{SM}}(p)=\epsilon_{\mathrm{SM}}(p)+\gamma+\mu$

and

$b_{\mathrm{DA}}(p)=\epsilon_{\mathrm{DA}}(p)+\gamma+\mu$,

where $\epsilon_{\mathrm{SM}}(p)$ and $\epsilon_{\mathrm{DA}}(p)$ are obtained through fits to the stochastic model and the deterministic approximation curves for the corresponding $p$-value, as we did for the case $p=0.4$ by fitting to the curves in Fig. 1(g) and (h).

It is possible to obtain an analytical approximate expression to estimate $b_{\mathrm{DA}}$ using the deterministic equations as follows. At the beginning of epidemic growth, when $X_{i} \ll 1$, we have that $X_{r}, X_{s i}$ and $X_{s r}$ are also $\ll 1$. So, we drop all the second order terms in $X_{i}, X_{r}, X_{s i}$ and $X_{s r}$, and also the terms $X_{i i}, X_{r r}, X_{i r}$. As $X_{s}=1-X_{i}-X_{r}$, we may write Eq. (7) as

$\frac{d X_{i}}{d t} \simeq-\gamma X_{i}-\mu X_{i}+p \beta X_{i}+\frac{q \beta X_{s i}}{4}$.

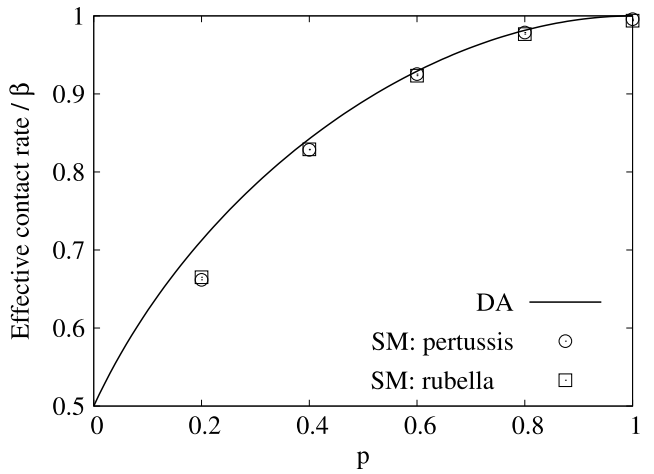

Fig. 2. Comparison of the stochastic model (SM) and the deterministic approximation (DA) predictions for the effective contact rate during the exponential growth in an epidemic as a function of $p$. Values are relative to the corresponding $\beta$-value for each disease. For the deterministic approximation $b_{\mathrm{DA}}^{*} / \beta$ is computed through Eq. (19). For the stochastic model, $b_{\mathrm{SM}}$ is obtained through Eq. (15) by fitting the exponential regime of the average fraction of infected individuals as a function of time (shown in Fig. $1(\mathrm{~g})$ and $(\mathrm{h})$ for the case $p=0.4)$.

If $X_{i}(t)$ presents an exponential behavior, $d X_{i} / d t$ should be proportional to $X_{i}$, so, we assume that in this exponential regime: $X_{s i} \simeq K X_{i}, K$ being a constant. Eq. (17) may now be written as:

$\frac{d X_{i}}{d t} \simeq-\gamma X_{i}-\mu X_{i}+\left(p+\frac{q K}{4}\right) \beta X_{i}$,

where $(p+q K / 4) \beta$ plays the same role as $\beta$ in the exponential growth when only global contacts are present. So, we define $b_{\mathrm{DA}}^{*}=$ $(p+q K / 4) \beta$.

Taking $X_{s i} \simeq K X_{i}$ and replacing it in Eqs. (7) and (10), we may obtain the value of $K$ and compute:

$b_{\mathrm{DA}}^{*} \simeq \frac{1}{4}\left(1+p+\sqrt{1+10 p-7 p^{2}}\right) \beta$.

For $p=0.4$, this equation gives the same value we obtained above for $b_{\mathrm{DA}}$ within the precision of the fit to the deterministic approximation curve in Fig. $1(\mathrm{~g})$.

An interesting point here is that Eq. (19) predicts that $b_{\mathrm{DA}}^{*} / \beta$ only depends on $p$ and not on the other parameters characterizing the disease transmission. In Fig. 2 we plot this relation and compare with $b_{\mathrm{SM}} / \beta$ obtained through the fits for different $p$-values for pertussis and rubella. The agreement between the deterministic approximation and the stochastic model is good, but it becomes worse for lower values of $p$. However, even for $p=0.2$, when the agreement of the stochastic model and the deterministic approximation is not so good $\left(b_{\mathrm{SM}}=0.529, b_{\mathrm{DA}}=0.57\right.$, for pertussis, and $b_{\mathrm{SM}}=0.259, b_{\mathrm{DA}}=0.277$, for rubella) $b_{\mathrm{SM}} / \beta$ verifies quite well the deterministic approximation prediction of being dependent only on $p$ for pertussis (0.661) and rubella (0.666). We mean that for low $p$, Eq. (19) does not approach $b_{S M}$ accurately, but $b_{\mathrm{SM}} / \beta$ seems to basically depend on $p$, as the deterministic approximation predicts.

\subsection{Stationary behavior}

For the stochastic model presented in Section 2 and a finite value of $N$, the only fixed point (stationary equilibrium) corresponds to the case where all the individuals on the lattice are susceptible. Due to the stochastic nature of the system, sooner or later a fluctuation leads the number of infected individuals to zero, and there is no process that produces new infected people if there are none. But for large values of $N$ (as the one taken in the present study) the system may fluctuate for a long time around a quasi-stationary state before extinction. The definition and properties of such a state have been addressed in other 

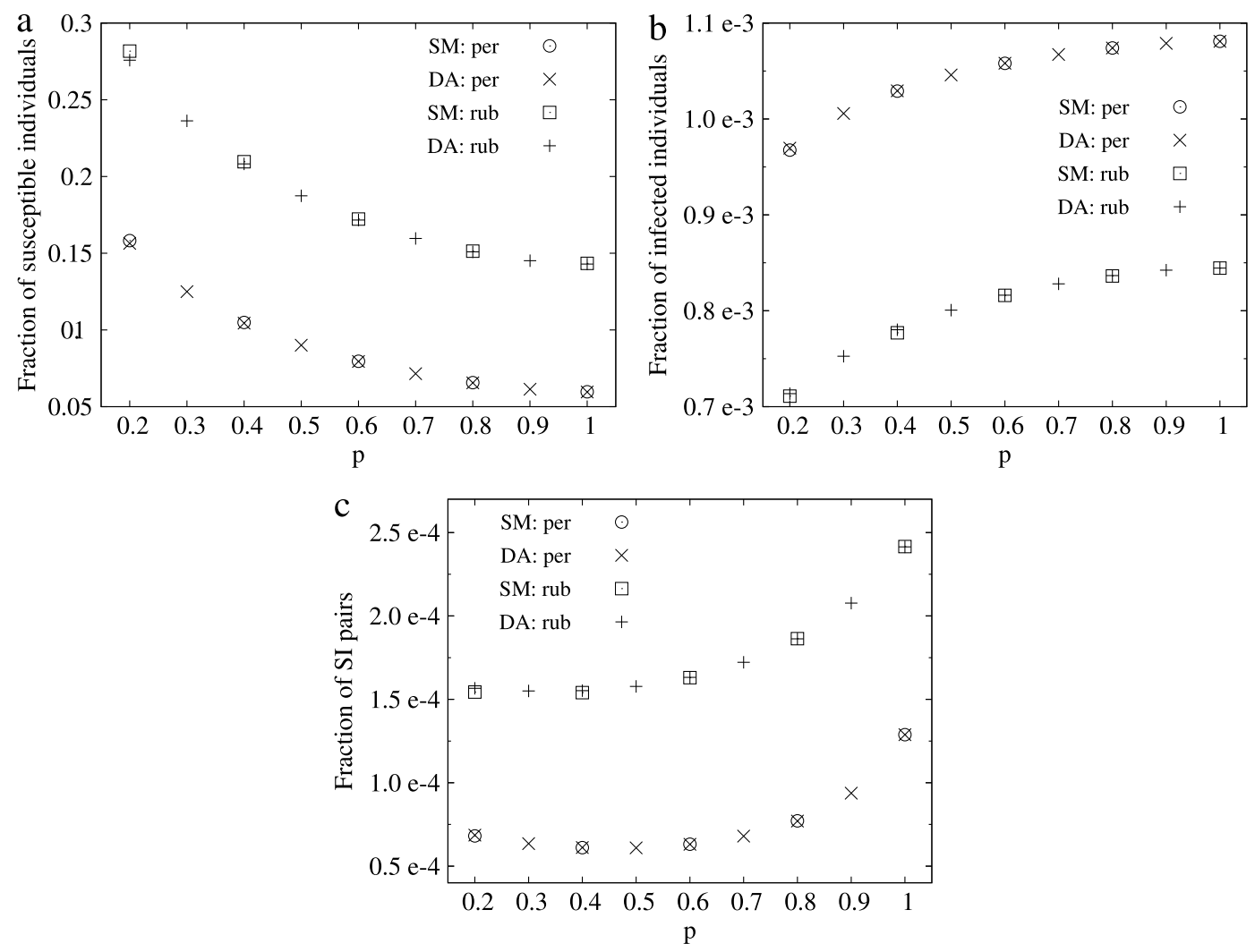

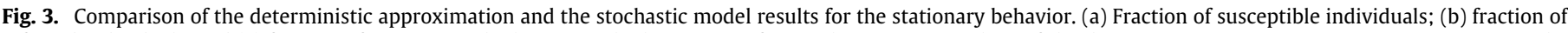

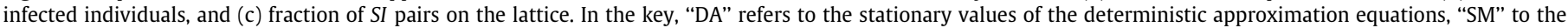
estimated average magnitudes in the stochastic model, "per" to pertussis and "rub" to rubella.

contexts from a mathematical point of view (Darroch and Seneta, 1967; Nåssel, 1999) or with empirical approaches (Martins de Oliveira and Dickman, 2005; Blanchet et al., 2012). In the present work, we adopt the empirical strategy developed by Dottori and Fabricius (2015), where the quasi-stationary state of the same stochastic model defined in Section 2 is studied. We define a time window $\left(t_{a}, t_{b}\right)$ and generate (for each system studied) more than 20,000 independent samples, keeping those that do not become extinct before $t_{b}$. We compute the averages of susceptible and infected individuals over the surviving samples and observe that in the $\left(t_{a}, t_{b}\right)$ interval they remain constant in time within a given precision. We take $t_{a}=20,000$ days and $t_{b}=40,000$ days; with these values the considered averages are independent of the initial conditions as $t_{a}$ is large enough so that correlations in the system may be established. For details of the definition of the quasi-stationary state and characterization of the fluctuations for the pertussis case, see Dottori and Fabricius (2015).

In the case of the deterministic approach, stationary values are defined as the values of the variables that make all the derivatives in Eqs. (6)-(14) equal to zero. They are obtained by numerically solving the equations from given initial conditions, until the unknowns are constant in time with a precision $10^{-5}$. As initial conditions we take, for $X_{s}, X_{i}$ and $X_{r}$, the values corresponding to the stationary solution of the SIR model and complete the remaining initial values as in the uncorrelated case. For example, $X_{s i}$ is initialized as $4 X_{S} X_{i}$ because it is twice the probability that a given pair is of type $S I$, whose value is $2 X_{S} X_{i}$ in the uncorrelated case.

In Fig. 3(a) and (b) we compare the deterministic approximation stationary values obtained for the fractions of susceptible and infected individuals for pertussis and rubella with the values of these observables averaged over the samples in the quasistationary state of the stochastic model. In Fig. 3(c), we make the same comparison for the fraction of SI pairs on the lattice. For both diseases, the deterministic approximation results show a close agreement with the stochastic model averaged magnitudes. The points corresponding to the stochastic model and the deterministic approximation in Fig. 3 are distinguished from each other only at low $p$ values, where the difference between the values calculated by the two methods is at most $2 \%$.

In Appendix, using the deterministic approximation, we analyze the stability of the system at the stationary values and find that the system is asymptotically stable for a wide range of the model parameters. Therefore, the deterministic approach proposed here could be applied to the study of the steady state for parameters corresponding to other infectious diseases. However, it is not obvious that the close agreement between the deterministic approximation and the stochastic model results found here will be obtained for other model parameters, in particular, if they lead to a greater presence of $S$ and I individuals in the system. The key point of the pair approximation (which describes the local infective contacts in the deterministic approximation) is to approach the probability of occurrence of a given triplet through Eq. (5). If the triplets that influence the dynamics of the local rate of infection, SSI and ISI, do not appear very often (as in the case of all the quasi-stationary states of the stochastic model studied in the present work), the pair approximation seems to work well enough in the deterministic approach. But for other parameters that involve a greater presence of $S$ and I individuals, it could be necessary to implement other schemes that go beyond the pair approximation to obtain a quantitative agreement with the stochastic model results. For example, we could implement schemes that explicitly include the triplets and approach the probability of the quadruplets, such as the triplet approximation used by Rozhnova and Nunes (2009) to study an SIRS model in random networks (see also Keeling and 

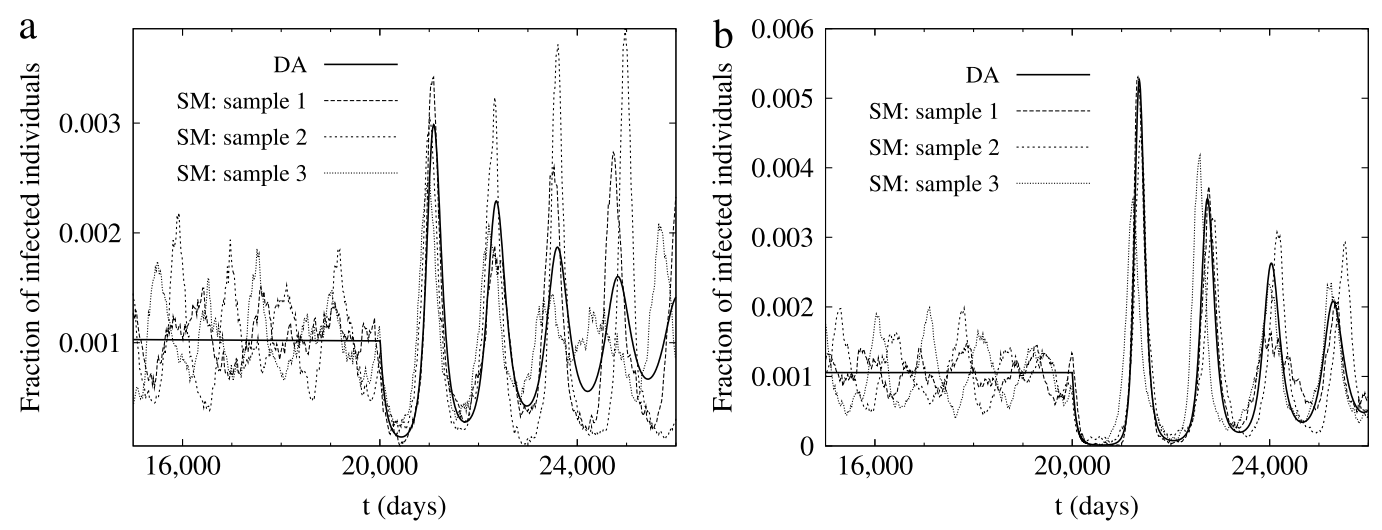

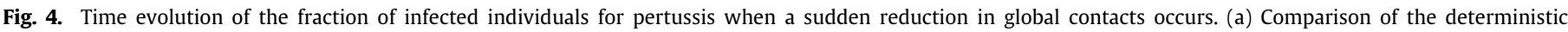

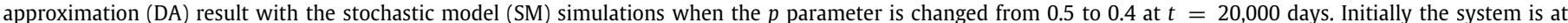

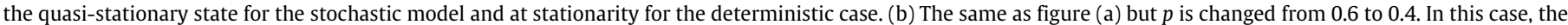

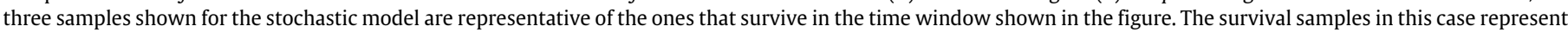
a fraction of $26 \%$ from all the generated samples.

Rohani, 2008 for other approaches that have been tried beyond the pair approximation).

\subsection{System response to a change in $p$}

\subsubsection{Comparison between the stochastic model and the deterministic approximation results}

We here study the ability of the deterministic approximation to describe the system response to a sudden change in the weight of global contacts represented in the model by the parameter $p$. We simulate disease transmission at the endemic state in a city where, at a given time, infective contacts among people change and become more local. Such a change could occur because of several circumstances that affect social activities. For example, in Argentina, during the 2009 pandemic flu, the authorities took preventive measures that included suspension of classes at school in July. The population was advised to stay at home whenever possible and to avoid crowded places. These measures aimed at reducing the possibility of an epidemic outbreak during the winter. Our purpose here is to simulate the consequences that this sudden reduction in the global contacts may have on the disease transmission of other infectious diseases that are at the endemic state.

We perform simulations with the stochastic model for parameters corresponding to pertussis in the prevaccine era and $p=0.5$. Once the quasi-stationary state is established, at a given time $(t=$ 20,000 days) we change the value of $p$ to 0.4 , which corresponds to a $20 \%$ reduction in global contacts. In Fig. 4(a) we compare the dynamical evolution of the system for three samples obtained from the stochastic model simulations and the deterministic approximation result. To obtain the deterministic approximation curve, as initial conditions we take the values corresponding to the stationary state for $p=0.5$ and at $t=20,000$ we change the value of $p$ to 0.4 .

We observe that the deterministic approximation captures the decrease in the fraction of infected individuals after lowering $p$ and the pronounced peaks observed in the following years. The perturbation at $t=20,000$ days puts in phase the oscillations for the different samples that become out of phase again from the third peak. The deterministic approximation also accurately reproduces the time elapsed between peaks. Oscillations are exponentially damped out in the deterministic approximation until the new stationary value is reached. In the case of the stochastic model, fluctuations may be different from sample to sample (Dottori and Fabricius, 2015), but as we have seen in Section 4.2, once the quasi-stationary state is reached, the fraction of infected individuals oscillates around a value similar to that predicted by the deterministic approximation.

We repeated this study for the case in which the system is initially at the quasi-stationary state corresponding to $p=0.6$ and at $t=20,000 p$ is changed to 0.4 . In this case, although the final state is the same, the reduction in global contacts is $33 \%$. In Fig. 4(b) it can be seen that after the perturbation, the system reaches a deep minimum followed by pronounced maxima much higher than in the previous case when the change in $p$ was $20 \%$ Again, the deterministic approach gives a good description of the observed effects. In this case, most of the samples obtained from the stochastic model simulations become extinct when the system is at the first and pronounced minimum. This is not surprising and could be inferred from the deterministic approximation result that predicts the number of infected individuals to be below 15 in the time interval $(20,400-20,800$ days) for the population considered here. Extinctions in the stochastic model simulations are thus expected, given the fluctuations in the number of infected individuals observed in the dynamic behavior of the system.

It is worth mentioning that a dynamic behavior similar to that observed in Fig. 4 has been found in a recent study of pertussis transmission performed with an age-structured deterministic model with nine epidemiological classes (Pesco et al., 2014). In that work, the authors proposed that a sudden change in contact rates among individuals could cause a dynamic effect with the presence of deep valleys followed by sharp maxima such as those recently observed in some US states. Here we obtain a similar behavior for the disease transmission as a consequence of reducing the relative importance of the global contacts in a much simpler epidemiological model.

\subsubsection{Further study with the deterministic approach}

In this section, our purpose is to explore with the deterministic approach some dynamical features of this problem that would be cumbersome to achieve with the stochastic model. We do not pretend to exhaust the analysis of this problem but to show the usefulness of the deterministic approach. We now introduce the dynamical variables to be studied. The system is initially at stationarity with $p=p_{i n}$. When the weight of the global contacts is reduced to $p_{f}$, the fraction of infected individuals is drastically reduced reaching a minimum value $X_{i}^{\min }$ and then, after several outbreaks, the system reaches the new stationary state corresponding to $p_{f}$. We call $T_{1}$ the time period between the first two outbreaks after the change in $p$. The period between successive outbreaks decreases approximating a limiting value $T_{\infty}$ when the system is infinitely close to the steady state. The $T_{\infty}$ value is 

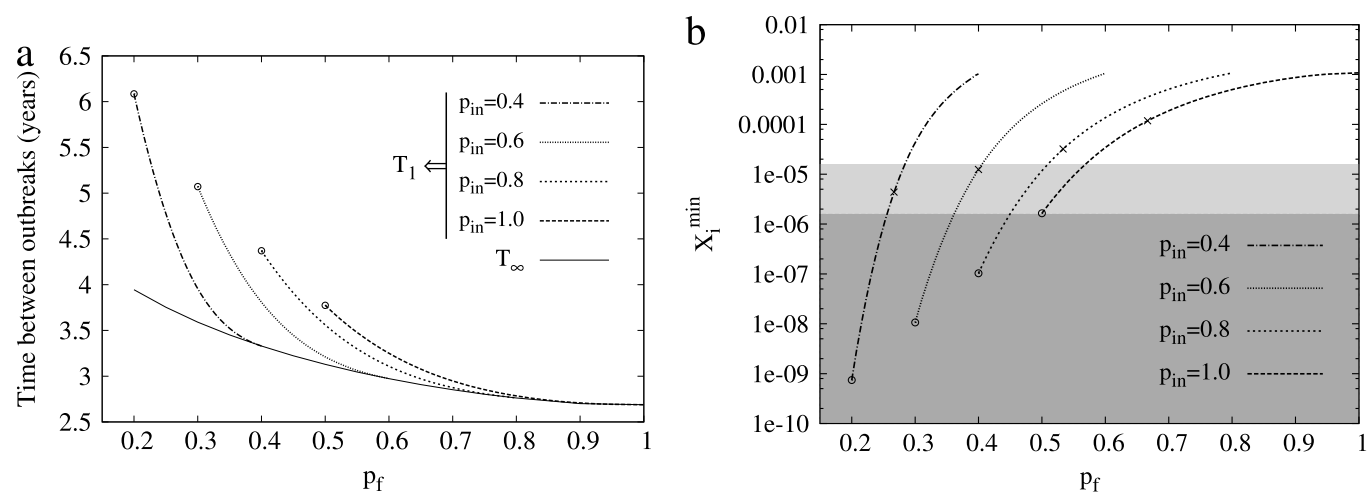

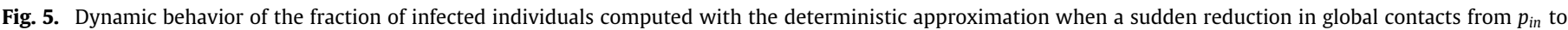

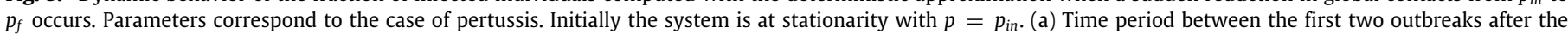

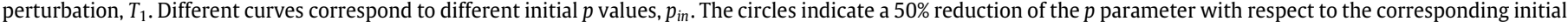

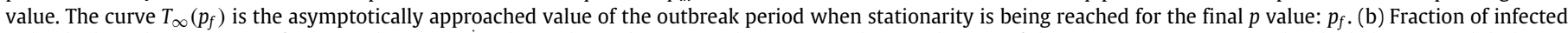

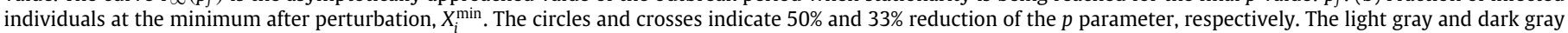
areas correspond to a number of infected individuals between 1 and 10 , and below 1 , respectively, for the 640,000 population considered in the present work.

also the period of the oscillations of infinitesimal amplitude that would be obtained by any slight perturbation of the new stationary state corresponding to $p_{f}$. Therefore, $T_{\infty}$ is a magnitude associated with the new stationary state only, while $T_{1}$ depends on both $p_{\text {in }}$ and $p_{f}$. We calculate with the deterministic approximation the magnitudes $T_{1}$ and $X_{i}^{\mathrm{min}}$ for four values of $p_{\text {in }}$ considering in each case a range of values for $p_{f}$ from 0.2 to 1 with a $p$-step of 0.001 . The results are presented in Fig. 5.

In Fig. 5(a), we show the curves $T_{1}\left(p_{f}\right)$ for different $p_{i n}$ values, and the curve $T_{\infty}\left(p_{f}\right)$. For a given $p_{i n}$ value, the larger the reduction in $p$ to the new value $p_{f}$, the larger the value of $T_{1}\left(p_{f}\right)$, and also the larger the difference between $T_{1}\left(p_{f}\right)$ and the asymptotic period $T_{\infty}\left(p_{f}\right)$. On the other hand, comparison among different $p_{\text {in }}$-curves shows that for the same relative reduction of $p$, the values of $T_{1}$ and $T_{1}-T_{\infty}$ are significantly larger for smaller values of $p_{i n}$. The approach of all the $T_{1}$-curves to the $T_{\infty}$-curve as $p_{f}$ increases may be understood as follows: for $p_{\text {in }} \simeq p_{f}$, the stationary state before the perturbation is in fact a slight alteration of the stationary state after the perturbation; then, when letting the system evolve with $p=p_{f}$, the system approaches stationarity from the beginning with small amplitude oscillations of period $T_{\infty}\left(p_{f}\right)$.

We now analyze the tendency to extinction of the system at the first minimum. We find this interesting because extinction is an exclusive feature of the stochastic model that cannot occur working with the deterministic approximation. However, the value of the fraction of infected individuals at the minimum, $X_{i}^{\mathrm{min}}$, is an indicator of the proximity to extinction in the stochastic model. For example, for a change in $p$ from 0.5 to 0.4 (Fig. 4(a)), out of 50,000 samples considered only $11 \%$ become extinct, while for a change in $p$ from 0.6 to 0.4 (Fig. 4(b)) $74 \%$ die out. This is clearly related to the fact that $X_{i}^{\text {min }}$ values obtained with the deterministic approximationare $1.410^{-4}$ and $1.310^{-5}$ for Fig. 4(a) and (b), which correspond (for the 640,000 population used for the stochastic model simulations) to having 90 and 8 individuals at the minimum, respectively. In Fig. 5(b), the $X_{i}^{\min }$ curves for the same changes of $p$ analyzed in Fig. 5(a) are shown. We notice that $X_{i}^{\min }$ values are sensitive to the change in the value of the $p$ parameter. To analyze the tendency of the system to extinction, we shaded an area (light gray) in the figure that includes the $X_{i}^{\text {min }}$ values corresponding to having between 1 and 10 infected individuals at the minimum. We assume that this area indicates a very probable "extinction zone", and that it is not reasonable to think that the corresponding simulations of the stochastic model in the zone below (dark gray area) will survive beyond the minimum. From the results presented in Fig. 5(b), the deterministic approximation suggests that for no initial value of $p$ the system supports a $50 \%$ reduction in its value (circles). While for a $33 \%$ reduction of $p$ (crosses), the tendency to extinction increases when $p_{\text {in }}$ diminishes, since the deterministic approximation predicts 75 , 20,8 and 3 infected individuals at the minimum for $p_{i n}=1,0.8$, 0.6 and 0.4 , respectively.

\section{Conclusions}

In this work, we propose a deterministic approach for an SIR-type epidemiological model that includes global and local contacts on a square lattice. When comparing our deterministic approach with simulations performed with the stochastic version of the model, we obtain a close agreement for qualitatively different scenarios of disease transmission and parameters corresponding to pertussis and rubella in the prevaccine era.

We study the epidemic spread in a fully susceptible population and find that stochastic fluctuations can produce a considerable variability of the triggering time for the epidemic. However, once the epidemic regime is established, the dynamical evolution of the system is only slightly influenced by stochastic effects and is closely described by the deterministic approximation. We find that the epidemic growth is characterized by an exponential regime with an exponent that decreases when the relative importance of the local contacts increases. Using the deterministic approach, we are able to find an explicit approximated expression for the exponent as a function of the model parameters.

At the quasi-stationary state of the system, the average fraction of susceptible and infected individuals, as well as the average fraction of susceptible-infected pairs is also closely approximated by the stationary values obtained using the deterministic approximation. Even though our study focuses on pertussis and rubella, other infectious diseases could be studied using the deterministic approximation, as the system is asymptotically stable around the stationary values for a wide range of model parameters.

We also consider, for the particular case of pertussis, the perturbation of the quasi-stationary state of the system by a sudden reduction of the global contacts. Such a change in the social behavior could be caused, for example, as a consequence of a health control measure that urges people to avoid public places. The fraction of infected individuals shows a dynamic behavior after the perturbation characterized by the presence of a deep minimum followed by a series of sharp and well-defined outbreaks. We find that this behavior is also qualitatively well reproduced by the deterministic approximation and, taking advantage of the much simpler computations involved, we characterize the period between outbreaks after the perturbation and the propensity of 


\begin{tabular}{|c|c|c|c|c|}
\hline $\begin{array}{c}\qquad \begin{array}{c}-p \beta X_{i}-\mu \\
p \beta X_{i} \\
4 \mu+U V W \\
U V(V / 2-W) \\
-U V^{2} / 2\end{array} \\
\text { where } U=3 q \beta / 8, V=\end{array}$ & $\begin{array}{c}-p \beta X_{s} \\
p \beta X_{s}-\gamma-\mu \\
-2 p \beta X_{s s} \\
p \beta\left(2 X_{s s}-X_{s i}\right)+4 \mu \\
p \beta X_{s i} \\
X_{s i} / X_{s}, W=X_{s s} / X_{s} .\end{array}$ & $\begin{array}{c}0 \\
0 \\
-2 p \beta X_{i}-U V-2 \mu \\
2 p \beta X_{i}+U V \\
0\end{array}$ & $\begin{array}{c}-q \beta / 4 \\
q \beta / 4 \\
-U W \\
U(W-V-2 / 3)-p \beta X_{i}-\gamma-2 \mu \\
p \beta X_{i}+U V+q \beta / 4\end{array}$ & $\left.\begin{array}{c}0 \\
0 \\
0 \\
0 \\
-2(\gamma+\mu)\end{array}\right)$ \\
\hline
\end{tabular}

Box I.

the system to extinction as a function of the relative reduction of global contacts. An interesting prediction obtained from the deterministic approximation study but concerning the stochastic simulations is the extinction of infected individuals at the first minimum after perturbation if the global contacts are reduced by $50 \%$. This prediction holds regardless of the weight of the global contacts at the initial state of the system before it is drastically reduced.

It would be valuable to increase the complexity of the model used in the present work in order to study pertussis transmission in the vaccine era. The introduction of pertussis vaccination in the model forces one to include new epidemiological classes that account for individuals that have partially (or totally) lost their immunity (Hethcote, 1997, 1999; Fabricius et al., 2013), as it is well known that immunity to pertussis conferred by vaccines is not lifelong (Wendelboe et al., 2005). On the basis of the deterministic approximation developed in Section 3, it would be straightforward to implement a deterministic approach to a stochastic model with local and global contacts that includes additional epidemiological classes. Stochastic simulations for a more complex model could be much more time-consuming, and a deterministic approximation would be a convenient alternative to study the problem.

\section{Acknowledgments}

This work was supported by Agencia Nacional de Promoción Científica y Tecnológica-ANCPyT grant PICT2010-0707. G.F. is member of the Scientific Career of Consejo Nacional de Investigaciones Científicas y Tecnológicas-CONICET (Argentina).

\section{Appendix}

In this Appendix we examine the stability of the deterministic equations under changes in the model parameters. For this purpose we first reduce the system of equations (6)-(14) by using (1)-(4), obtaining

$$
\begin{aligned}
\frac{d X_{s}}{d t}= & -p \beta X_{s} X_{i}-\frac{q \beta X_{s i}}{4}-\mu X_{s}+\mu, \\
\frac{d X_{i}}{d t}= & -(\gamma+\mu) X_{i}+p \beta X_{s} X_{i}+\frac{q \beta X_{s i}}{4}, \\
\frac{d X_{s s}}{d t}= & -2 p \beta X_{i} X_{s s}-\frac{3 q \beta X_{s i} X_{s s}}{8 X_{s}}+4 \mu X_{s}-2 \mu X_{s s}, \\
\frac{d X_{s i}}{d t}= & -p \beta X_{i} X_{s i}-3 q \beta\left(\frac{\left(X_{s i}\right)^{2}}{16 X_{s}}-\frac{X_{s i} X_{s s}}{8 X_{s}}\right)+2 p \beta X_{i} X_{s s} \\
& -\left(\frac{q \beta}{4}+\gamma+2 \mu\right) X_{s i}+4 \mu X_{i},
\end{aligned}
$$

$\frac{d X_{i i}}{d t}=-2(\mu+\gamma) X_{i i}+p \beta X_{i} X_{s i}+q \beta\left(\frac{3\left(X_{s i}\right)^{2}}{16 X_{s}}+\frac{X_{s i}}{4}\right)$.

We study the stability of this autonomous system by analyzing the Jacobian matrix of the linearized system (given in Box I).

We perform a numerical eigenanalysis of the preceding matrix at the stationary values, concluding that the system is asymptotically stable in the range of $p$ included in this study $(0.2 \leq p \leq 1)$ for a wide range of the parameters $(\gamma / \mu, \beta / \mu)$ that includes most of infectious diseases in the pre-vaccine era: $2 \leq \beta / \gamma \leq 30,0.0005 \leq \mu / \gamma \leq 0.002$ (Anderson and May, 1991; Simöes et al., 2008).

\section{References}

Anderson, R., May, R., 1991. Infectious Diseases of Humans: Dynamics and Control. Oxford University Press, Oxford, UK.

Aparicio, J., Pascual, M., 2007. Building epidemiological models from $R_{0}$ : an implicit treatment of transmission in networks. Proc. R. Soc. B 274, 505-512.

Ball, F., Mollison, D., Scalia-Tomba, G., 1997. Epidemics with two levels of mixing. Ann. Appl. Probab. 7, 46-89.

Ball, F., Sirl, D., Trapman, P., 2010. Analysis of a stochastic SIR epidemics on a random network incorporating household structure. Math. Biosci. 224, 53-73.

Bartlett, M., 1956. Deterministic and stochastic models for recurrent epidemics. In: Proc. Third Berkeley Symp. on Math. Statist. and Prob., Vol. 4. Univ. of Calif. Press, pp. 81-109.

Bauch, C., Earn, D., 2003. Transients and attractors in Epidemics. Proc. R. Soc. Lond. Ser. B 270, 1573-1578.

Blanchet, J., Glynn, P., Zheng, S., 2012. Empirical analysis of a stochastic approximation algorithm for computing quasi-stationary distributions. In: EVOLVEA Bridge between Probability, Set Oriented Numerics, and Evolutionary Computation II. In: Advances in Intelligent Systems and Computing, vol. 175. pp. 19-37.

Darroch, J., Seneta, E., 1967. On quasi-stationary distributions in absorbing continuous-time finite Markov chains. J. Appl. Probab. 4 (1), 192-196.

Diekmann, O., Heesterbeek, H., Britto, T., 2013. Mathematical Tools for Understanding Infectious Disease Dynamics. Princeton University Press, Princeton.

Dottori, M., Fabricius, G., 2015. SIR model on a dynamical network and the endemic state of an infection disease. Physica A 434, 25-35.

Ellner, S., 2001. Pair approximation for lattice models with multiple interaction scales. J. Theoret. Biol. 210 (4), 435-447.

Eubank, S., et al., 2004. Modelling disease outbreaks in realistic urban social networks. Nature 429, 180-184.

Fabricius, G., Bergero, P., Ormazabal, M., Maltz, A., Hozbor, D., 2013. Modelling pertussis transmission to evaluate the efectiveness of an adolescent booster in Argentina. Epidemiol. Infect. 141, 718-734.

Gillespie, D., 1976. A general method for numerically simulating the stochastic time evolution of coupled chemical reactions. J. Comput. Phys. 22, 403-434

Granich, R., Gilks, C., Dye, C., De Cock, K., Williams, B., 2009. Universal voluntary HIV testing with inmediate antiretroviral therapy as a strategy for elimination of HIV transmission: a mathematical model. Lancet 373, 48-57.

Heesterbeek, H., et al., 2015. Modelling infectious disease dynamics in the complex landscape of global health. Science 347, aaa4339. http://dx.doi.org/10.1126/ science.aaa4339.

Hethcote, H., 1997. An age-structured model for pertussis transmission. Math. Biosci. 145, 89-136.

Hethcote, H., 1999. Simulations of pertussis epidemiology in the United States: effects of adult booster vaccinations. Math. Biosci. 158, 47-73.

Joo, J., Lebowitz, J., 2004. Pair approximation of the stochastic susceptible-infectedrecovered-susceptible epidemic model on the hypercubic lattice. Phys. Rev. E 70, 036114.

Keeling, M., Eames, K., 2003. Networks and epidemic models. J. R. Soc. Interface 2, 297-307.

Keeling, M., Rohani, P., 2008. Modeling Infectious Diseases in Humans and Animals. Princeton University Press, Princeton.

Keeling, M., Rohani, P., Grenfell, B., 2001. Seasonnaly forced disease dynamics explored as switching between attractors. Physica D 148, 317-335.

Kermack, W., McKendrick, A., 1927. A contribution to the mathematical theory of epidemics. Proc. R. Soc. Lond. Ser. A 115, 700-721.

Martins de Oliveira, M., Dickman, R., 2005. How to simulate the quasistationary state. Phys. Rev. E 71, 016129

Nåssel, I., 1999. On the time to extinction in recurrent epidemics. J. R. Stat. Soc. Ser, B Stat. Methodol. 61, 309-330.

Pastor-Satorras, R., Castellano, C., Van Mieghem, P., Vespignani, A., 2015. Epidemic processes in complex networks. Rev. Modern Phys. 87, 925.

Pesco, P., Bergero, P., Fabricius, G., Hozbor, D., 2014. Modelling the effect of changes in vaccine efectiveness and transmission contact rates on pertussis epidemiology. Epidemics 7, 13-21.

Rohani, P., Earn, D., Grenfell, B., 1999. Opposite patterns of synchrony in sympatric disease metapopulations. Science 286, 968-971. 
Rozhnova, G., Nunes, A., 2009. Cluster approximations for infection dynamics on random networks. Phys. Rev. E 80, 051915.

Rozhnova, G., Nunes, A., 2012. Modelling the long term dynamics of prevaccination pertussis. J. R. Soc. Interface 9, 2959-2970.

Simöes, M., Telo da Gama, M., Nunes, A., 2008. Stochastic fluctuations in epidemics on networks. J. R. Soc. Interface 5, 555-566.

Souza, D., Tomé, T., 2010. Stochastic lattice gas model describing the dynamics of the SIRS epidemic process. Physica A 389, 1142-1150.
Verdasca, J., Telo da Gama, M., Nunes, A., Bernardino, N., Pacheco, J., Gomes, M., 2005. Recurrent epidemics in small world networks. J. Theoret. Biol. 233, 553-561.

Watts, D., Strogatz, S., 1998. Collective dynamics of "small world" networks. Nature 393, 440-442.

Wendelboe, A., Van Rie, A., Salmasso, S., Englund, J., 2005. Duration of inmunity against pertussis after natural infection or vaccination. Pediatr. Infect. Dis. J. 24, S5-S98. 